The Detection and Control of Garden Pests

By G. Fox Wilson. (Agricultural and Horticultural Series.) Pp. $194+54$ plates. (London: Crosby Lockwood and Son, Ltd., 1947.) 12s, $6 d$. net.

AOOD sound book on pests of the garden has long been overdue, and Mr. Fox Wilson's effort certainly fills the gap admirably. Very often insect damage to cultivated plants is uncommonly difficult to identify. Unless it can be recognized in its early stages and measures of repression put into force, it is usually "too late to mend". The book is an expanded and revised version of the author's serial articles entitled "Symptoms of Pest Attack on Plants" published in the Gardener's Chronicle during 1932-36. The basis of the book is, therefore, laid on diagnosing pest injuries, and each chapter deals with the insects or other pests of a separate part of plants. Thus Chapter 3 deals with roots, and here the reader will find an account of the injuries inflicted by all the root-enemies that are likely to be encountered. Other chapters deal with stems and shoots, flowers, seeds, and so on. There is no doubt that this method of treating the subject facilitates quick identification better than any method of grouping the different pests under their natural orders or their specific plants.

The measures advocated for the control of the various pests are the best that present-day knowledge can offer-some are completely effective, others partially so. The photographic illustrations of damaged plants are a useful aid together with those of individual pests. While the nomenclature used for different pests is not always very modern, little confusion will result. In fact, it will prove an advantage to the general reader for the reason that it will facilitate reference to works of earlier date, since in these works the older names are current.

The author has done a real service in writing this book at a time when we need to produce and conserve so much in the vegetable kingdom.

A. D. I.

\section{The Adjustment and Testing of Telescope} Objectives

By H. Dennis Taylor. Fourth edition. Pp. viii+95. (Newcastle-upon-Tyne: Sir Howard Grubb, Parsons and Co., 1946.) 17s. $6 d$.

$\mathrm{T}$ HOUGH the first edition of this work was published more than fifty years ago, in 1891, it still has value as a standard work on the adjustment and testing of telescope objectives. The firm of Sir Howard Grubb, Parsons and Co., which has taken over the astronomical interests of Messrs. Cooke, Troughton and Simms, has performed a useful service in publishing a new edition. This edition follows the text of the third edition, published in 1921. The new edition would have had a greatly increased value, however, if the opportunity had been taken to revise the material thoroughly and to make additions to bring it up to date. New methods of testing objectives are available and might well have been included. The introdtaction to the volume refers to the refracting telescope as "the most convenient and generally reliable form of telescope"; but since this was written the reflecting telescope has become much more favoured. All large modern telescopes are necessarily reflectors, and reflectors are much used by amateurs because, for a given aperture, a reflector is less costly than a refractor and also because mirror making is within the ability of many amateurs. It would have been valuable to have in. cluded an account of methods of testing mirrorsspherical, paraboloidal and hyperboloidal. Other material could with advantage now be omitted as not strictly pertinent to the subject of the book. At some future date, a new edition is likely to be required, and it is suggested that the opportunity might then be taken to make a complete revision.

\section{I fondamenti scientifici della astronomia}

Per Prof. G. Armellini. Pp. xv +320 . Ulrico Hoepli, 1947.) 650 lire.

$7 \mathrm{HE}$ aim of the author in writing this book was to fill a gap in Italian scientific literature by presenting a logical mathematical account of the foundations of that part of astronomy which is concerned with the study of the movements of the celestial bodies, and with the calculation of their distances and of their dimensions. The book is in. tended primarily for the students attending courses in astronomy at Italian universities, and the knowledge of mathematics required does not go beyond simple applications of the differential calculus. In addition to the ground usually covered in elementary textbooks on spherical astronomy, the author deals with the calculation of an orbit from three observations, with the determination of the solar motion, with the rotation of the galaxy and the determination of its dimensions and mass, with elementary stellar statisties and dynamics, with the distribution of the external galaxies and with the curvature of space. Most of the subjects dealt with are necessarily discussed only in an introductory manner. The student who masters this book will, however, have a sufficient grounding to enable him to proceed to the study of more advanced text-books. The author plans to follow this work with a further volume on the scientific foundations of astrophysics, which will be concerned with stellar photometry, astronomical spectroscopy, and the physics of the sun, planets, stars and nebulæ.

H. S. J.

\section{Native Peoples of the Pacific World}

By Prof. Felix M. Keesing. (Pacific World Series.) Pp. $x v+144+32$ plates. (New York: The Macmillan Company; London: Macmillan and Co., Ltd., 1946.) 15s. net.

GOR most people there is a strange glamour about the South Sea Islands and their inhabitants, due in part, no doubt, to the works of such visitors as R. L. Stevenson and Gauguin. But few people know anything accurately either about the islands themselves or about the various peoples who live there, their material culture, customs, etc.

This book, written mainly for American soldiers during the War, sets out to supply this need, and it has the great advantage of being written by a specialist; Prof. F. M. Keesing was born in Malaya, brought up in New Zealand, and has been professor of anthropology in the University of Hawaii. He is at present professor of anthropology in Stanford University, California. After a general chapter on the geography of the South Sea Islands, the Polynesians, Micronesians and Melanesians are in turn briefly described, such matters as languages, types of government, methods of livelihood, home conditions and social customs being mentioned. Finally, there is a chapter on religion. The whole is profusely illustrated and the work is very readable. Unfor. tunately, there is no bibliography; but a beginner interested in these far-away peoples will here find an excellent introduction to the subject. M. C. B. 